\title{
Percepción de la calidad de vida desde los principios de la complejidad
}

\section{Perception about the quality of life based on the principles of complexity}

\author{
Harold Guevara'; Antonio Domínguez ${ }^{\mathrm{II}}$; Magaly Ortunio ${ }^{\mathrm{III}}$. Daniella \\ Padrón ${ }^{\mathrm{IV}}$; Rosa Cardozov \\ IMédico Ocupacional. Profesor Asociado. Departamento de Salud Pública. \\ Universidad de Carabobo. Valencia, Venezuela. \\ IIDoctor en Ciencias Médicas. Profesor Titular. Departamento de Salud Pública. \\ Universidad de Carabobo. Valencia, Venezuela.

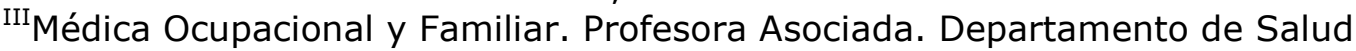 \\ Pública. Universidad de Carabobo. Valencia, Venezuela. \\ ${ }^{\mathrm{IV}}$ Doctora en Ciencias Sociales. Profesora Agregada. Departamento de Salud \\ Pública. Universidad de Carabobo. Valencia, Venezuela. \\ $\vee$ Médica Familiar. Profesora Asociada. Departamento de Salud Pública. Universidad \\ de Carabobo. Valencia, Venezuela.
}

\begin{abstract}
RESUMEN
La calidad de vida es objeto de interés de diferentes disciplinas. Indagar acerca de que los ciudadanos vivan bien tiene que ver con alcanzar el desarrollo de ciertas capacidades humanas que les permita llevar una vida plena. Se hace una revisión de las diferentes concepciones que hay sobre la calidad de vida tomando como referencia autores con diferentes enfoques filosóficos y epistemológicos. Se incluyen las posturas utilitaristas, la ética de las capacidades, la bioética y una visión desde la complejidad. Se intenta resaltar que la Calidad de vida es un área que merece atención por las facetas que abarca, por su interdimensionalidad y por la incertidumbre que lleva implícita, por lo que se demanda su abordaje desde el paradigma epistemológico de la complejidad.
\end{abstract}

Palabras clave: Calidad de vida, paradigma de la complejidad. 


\begin{abstract}
The quality of life is the object of interest of several disciplines. Making inquiries about the possibilities of the people to live well has to do with developing some human capabilities that allow them to enjoy full life. A review was made of the various conceptions about quality of life, taking several philosophic and epistemological approaches as reference. Utilitarian standpoints, the ethics of capacities, bioethics and vision based on complexity were included. It was intended to stress that the quality of life is an area deserving attention because of the facets that it embraces, its inter-dimensionality and the uncertainty inherent to it. Hence, this area requires to be approached from the epistemological paradigm of complexity.
\end{abstract}

Key words: Quality of life, paradigm of complexity.

\title{
INTRODUCCIÓN
}

La calidad de vida involucra el nivel de florecimiento humano existente en una sociedad, resulta área de interés para cualquier disciplina de las ciencias sociales que intente medir o evaluar cómo le va a la gente. Al considerarla es necesario no solo identificar la cantidad de dinero que tienen las personas, sino también saber de su expectativa de vida, su salud, servicios médicos a que pueden acceder, disponibilidad y calidad de su educación, trabajo, derechos que tienen y los que efectivamente les son respetados, privilegios legales y políticos que disfrutan, cómo están estructuradas las relaciones familiares y de género, y cómo la sociedad les permite imaginar, maravillarse y sentir emociones como el amor y la gratitud. ${ }^{1}$

La Organización Mundial de la Salud en 1994 propuso una definición para la calidad de vida:

Percepción del individuo sobre su posición en la vida, en el contexto de la cultura y sistema de valores en el cual él vive, y en relación con sus objetivos, expectativas, estándares e intereses. ${ }^{2,3}$

En este artículo se hace una revisión de las diferentes concepciones que hay sobre calidad de vida.

\section{CALIDAD DE VIDA Y FELICIDAD}

Algunos sostienen que la calidad de vida se debe medir en términos de utilidad, ya sea esta felicidad o satisfacción de deseos. Epicuro dividió las necesidades humanas en tres clases: naturales y necesarias (alimento y vestido), son relativamente fáciles de satisfacer; naturales pero no necesarias, (satisfacción sexual por ejemplo); y las que no son naturales ni necesarias, (lujo, abundancia, fausto y esplendor), siendo su satisfacción muy difícil. ${ }^{4}$ Sin embargo, dependiendo de la sociedad y cultura del colectivo, en algunos casos se observan viviendas muy precarias en situación de riesgo, con habitantes que no teniendo ni consumiendo lo necesario para subsistir, poseen lujosos equipos electrónicos, no "naturales" ni "necesarios". Quizás, intuitivamente, aplican los preceptos de Keyes $K$, de disfrutar 
de la vida...a pesar de todo. ${ }^{5}$ La distribución de la calidad de vida se considera para identificar categorías sociales que necesitan un especial cuidado como guía de la política social, inspirándose en el credo utilitarista de que el "fin último" de la política debería ser promover "la mayor felicidad para el mayor número de personas". Esta filosofía está en la base ideológica del estado de bienestar del siglo $\mathrm{xX}$, propulsor de la investigación en calidad de vida. ${ }^{6}$

El enfoque utilitarista queda sujeto a controversia, para Bueno, ${ }^{7}$ dicha controversia remite a la filosofía de la ciencia, la política del conocimiento, el modelo de sociedad y el concepto de ser humano como actor individual y social. Como puede apreciarse, la definición de calidad de vida humana implica necesariamente una antropología filosófica. Este autor hace un enfoque dialéctico: calidad individual frente a calidad colectiva. ¿Y a qué se le llama individual o colectivo? Carl Jung desarrolló una teoría acerca del inconsciente colectivo. Establece que existe un lenguaje común a los seres humanos de todos los tiempos y lugares del mundo, que serían representaciones simbólicas primitivas a través de las cuales se expresa un contenido de la psiquis que está más allá de la razón. ${ }^{8}$ Es innato, hereditario y ligado a los instintos.

\section{CALIDAD DE VIDA Y ÉTICA DE LAS CAPACIDADES}

Es posible considerar la vida de una persona como una combinación de funcionamientos, en los que un ser humano puede tener interés o se puede ver realizado. La Calidad de vida está íntimamente vinculada a las capacidades de una persona, es decir, a la libertad que cada uno tiene de elegir la clase de vida que quiere para sí. ${ }^{1}$ Para explicitar las bases filosóficas de la ética de las capacidades, $\mathrm{Sen}^{9}$ recurre a Aristóteles: "las relaciones conceptuales más importantes parecen ser las vinculadas con la noción aristotélica del bien humano". Debe establecerse primero la función del hombre y luego explorar la vida en el sentido de actividad. Nussbaum citada por Conill $J,{ }^{10}$ postula "una lista de funcionamientos que constituyen una buena vida humana" y una filosofía esencialista, según la cual la vida humana tiene ciertos rasgos centrales definitorios. ${ }^{11,12}$

Sin duda, se debe tener una concepción integral del ser humano, en el que confluyen lo biológico, psicológico, social, ético, político, cultural, histórico, económico, espiritual y lo estético, sin que a priori ningún aspecto prevalezca para la Calidad de vida, si bien para algunos la clave puede estar en lo económico, otros consideran que lo cardinal está en el ser y el hacer, en la capacidad de elegir ${ }^{1}$ el logro de buenos fines a través de buenos medios. Es decir, la integración de la reflexión y la acción, en vista que entender el por qué de algo hace más fácil el hacer frente a el cómo. ${ }^{13}$

Desde su perspectiva, Nussbaum propone una lista de capacidades humanas "esenciales", estructuradas en dos umbrales: a) un primer umbral compuesto por las funciones humanas básicas, por debajo de las cuales una vida estaría tan empobrecida que no sería humana, b) sobre este umbral, uno superior -de corte ético- debajo del cual estas funciones están disponibles en forma tan reducida que la existencia no se puede asumir como una buena vida humana.

Kohlberg y su equipo, citado por López, ${ }^{14}$ respaldan una ética de la justicia, que propone pasar del nivel preconvencional (lógica hedonista-instrumental) al convencional, que postula la autonomía como criterio, así como cambiar desde un enfoque instrumental hacia uno de derechos. El nivel posconvencional, se caracteriza por la pretensión universal y por el enfoque de los principios abstractos: el sujeto es capaz de ponerse en los zapatos del otro para decidir, y asumir como 
suyas las reglas morales, legales y los principios éticos universales para defender los derechos éticos de toda la humanidad y respetar la persona en sí misma. ${ }^{15}$

El umbral superior de Nussbaum es el que importa al abordar la política pública. Buscar que los ciudadanos vivan bien tiene que ver con desarrollar capacidades que se encuentran en el segundo umbral. Poseer estas capacidades es propio del ser humano, desarrollarlas es llevar una vida plena. ${ }^{16,17}$ Sen, ${ }^{18}$ propone la lista de capacidades como algo más bien contextual y menos rígido.

Desde mediados del siglo XX y comenzando el XXI, se está dando paso a rupturas por la desdogmatización de las teorías de la modernidad, enfatizando el espacio pluriparadigmático de la época. Rivero, ${ }^{19}$ destaca la necesidad de redefinir la ambigüedad marxista economicismo/voluntarismo en búsqueda de la conjunción a partir de un giro ético-político, para mostrar matices que amplían el pensamiento marxista como transdisciplinario, con posibilidad de dialogar en una perspectiva de pensamiento complejo. El enfoque de las capacidades coloca las decisiones acerca de una vida mejor del lado de los individuos, no así las condiciones de vida (colectivas). En la Constitución de la República Bolivariana de Venezuela se reivindica la calidad de vida como condiciones de vida, tanto como política social como política pública en salud.

\section{CALIDAD DE VIDA Y BIOÉTICA}

La calidad de vida está profundamente condicionada por la cultura, y depende estrictamente del conjunto de valores de los individuos y de los grupos sociales. Para Gracia, ${ }^{20}$ la calidad de vida se estructura en dos niveles, uno público y uno privado. Cada individuo define individual y socialmente su sistema de valores, su concepto de vida, de calidad y de felicidad. Desde ahí establece el propio proyecto de vida. Esta es la "ética de máximos", expresada en los principios de autonomía y beneficencia, la cual es deseable pero no exigible. Al mismo tiempo, los miembros de una sociedad deben contratar un conjunto de valores que sean respetados por todos, aun coactivamente. De allí que la "ética de mínimos", (ética pública), asume la definición general de calidad de vida que realiza la sociedad expresada en forma de leyes, sustentada en los principios de no maleficencia y de justicia. ${ }^{21}$

De tal manera que en la calidad de vida de los seres humanos están implícitos componentes personales y gregarios, cuya armonización es responsabilidad de todos como sujetos morales situados en y hermanados con el mundo. ${ }^{22}$ Por su parte, Boff ${ }^{23}$ postula lo que llama el "buen vivir", una visión integradora del ser humano, inmerso en la gran comunidad terrenal, que incluye además al aire, el agua, los suelos, las montañas, los árboles y los animales; es estar en profunda comunión con la Pachamama (Tierra), con las energías del Universo y con Dios, ejerciendo la simplicidad voluntaria. La mejor manera de alcanzarla es reducir intencionalmente las actividades vitales a sus elementos básicos, supone descargar la vida de todo lo que está de más, dejando de lado las distracciones que alejan aq todos de esa verdadera calidad de vida que se puede denominar plenitud. ${ }^{24}$

\section{CALIDAD DE VIDA DESDE LA COMPLEJIDAD}

La calidad de vida es un constructo social que surge en un marco de rápidos y continuos cambios sociales, que dirigen una incierta transición desde una sociedad industrial a una postindustrial, que puede situarse en el inicio de la década de los 70 del siglo $\mathrm{xx} .{ }^{25}$

Precisamente el concepto de calidad de vida ayuda a retomar la perspectiva del sujeto, superando y envolviendo el concepto de bienestar. Por ello resulta difícil 
acotarla, en vista que se construye socialmente como una representación que un colectivo puede tener sobre su propia vida. De ahí, la necesidad de profundizar en los análisis de las experiencias subjetivas y en los procesos que implican el desarrollo de la identidad social, destacando en la calidad de vida la espiritualidad, ya que el bienestar pleno es posible en los marcos de una vida dotada del sentido que ofrece el sistema de valores que el ser humano porta en su contexto social y cultural. ${ }^{26}$

La satisfacción óptima de las necesidades y por ende la consecución de altas cotas de calidad de vida es preciso buscarlas donde lo subjetivo y lo objetivo se funden, con base en una concepción sistémica y compleja de la realidad, en la que la idea de proceso permite entender la calidad de vida como una realidad dinámica, abierta y continuamente emergente. ${ }^{25}$

Los componentes de la calidad de vida se muestran como una realidad en la que hay muchos elementos emergentes que pueden interaccionar y retroaccionar en forma sinérgica, antagónica o complementaria para generarla y modificarla constantemente, tanto en la dimensión individual como en la colectiva, con base en los principios: sistémico (la realidad es unidad múltiple integrada por subsistemas en interacción constante), dialógico (la realidad configurada como síntesis dinámica e integradora de contrarios que se interconectan y se complementan), hologramático (el todo está inmerso en la parte y la parte es esencial y se comprende en tanto se encuentra inmersa en el todo), de recursividad (las causas generan efectos que a su vez retroaccionan sobre estas y autoorganizan el todo), de lo caótico (el orden tiene un caos implícito, que a su vez es creador de orden gracias a la autopoiesis), de lo impreciso e inacabado del conocimiento que sustentan la matriz epistémica de la Complejidad y el pensar sistémico. ${ }^{27-35}$ De allí que comprender la calidad de vida requiere la búsqueda continua de un equilibrio dinámico entre el conocimiento intuitivo y el conocimiento racional, lo cual choca con la racionalidad científica newtoniana-cartesiana tradicional propia de la civilización occidental.

Según la teoría general de sistemas el mundo se concibe con base en la interrelación e interdependencia de todos sus fenómenos; un sistema es una unidad integrada cuyas propiedades no pueden reducirse a las de sus partes, cada sistema está organizado formando estructuras poliniveladas, comprendiendo cada nivel un grupo de subsistemas que forman una unidad respecto a sus partes y una parte respecto a una unidad mayor. ${ }^{33-36}$ Por lo que cada ser humano es un "holón", un subsistema que es a la vez una unidad y parte de otra, poniendo de relieve la necesidad de conjugar y complementar la integración y la autoafirmación, de forma de lograr un equilibrio y que el sistema sea flexible y abierto al cambio, por el contrario, un comportamiento predominantemente agresivo y competitivo, podría condicionar un deterioro sostenido de la calidad de vida.

Según Capra "las teorías científicas jamás podrán proporcionar una descripción completa y definitiva de la realidad: siempre serán una aproximación a la verdadera naturaleza de las cosas", ${ }^{36}$ en tanto la realidad sea concebida y percibida con una visión sistémica, no lineal e inmersa en la incertidumbre, como es el caso de la calidad de vida vista desde la Complejidad.

\section{PARA PROSEGUIR LA BÚSQUEDA}

La calidad de vida es un constructo interdimensional, polinivelado, complejo y sistémico, a cuyo abordaje integrales posible aproximarse haciendo uso de los principios del paradigma de la complejidad, a los fines de evitar el reduccionismo 
del pensamiento lineal y percibir su realidad dinámica en toda su riqueza, incluyendo su incertidumbre inherente.

\section{REFERENCIAS BIBLIOGRÁFICAS}

1. Nussbaum M, Sen A. La Calidad de vida. México: Fondo de Cultura Económica; 1998.

2. Baldi G, García E. Calidad de vida y medio ambiente. La psicología ambiental. Universidades [sitio en Internet]. 2005 Jul-Dic [citado Dic 2009];30. Disponible en: http://www.udual.org/CIDU/Revista/30/Rev30.pdf

3. Lolas F. Discursos y narrativas en medicina: origen, ejemplos, comentarios. En: Lolas $F$, editor. Más allá del cuerpo. La construcción narrativa de la salud. Santiago de Chile: Editorial Andrés Bello; 1997. p. 159-62.

4. Shopenhauer A. Arte del buen vivir. 19na ed. Madrid: Editorial EDAF, S.A.; 2006.

5. Keyes K. Hacia la expansión de la conciencia: el camino a la felicidad a través del amor vivo. Madrid: Editorial EDAF, D.L.; 1992.

6. Ovalle O, Martínez J. La Calidad de vida y la felicidad. En: Contribuciones a la Economía [sitio en Internet] Dic 2006 [citado Dic 2009]. Disponible en: http://www.eumed.net/ce/2006/oojm.htm

7. Bueno S. Calidad de vida humana como criterio epistemológico. En: Educación, administración y Calidad de vida, Aula XXI. Editorial Santillana, Argentina [sitio en Internet]. 1990 [citado Dic 2009]. Disponible en:

http://www.santillana.com.ar/02/xtextos/0301.asp?level=\&mat=eteorico $\% 20 \&$ sec $=1 \#$ aca

8. Jung CG. Recuerdos, Sueños, Pensamientos. 7ma ed. Barcelona: Seix Barral; 2005.

9. Sen A. Capacidad y Bienestar. En: Nussbaum M, Sen A, editores. La Calidad de vida. México: Fondo de Cultura Económica; 1998. p. 74-5.

10. Conill J. Nueva propuesta de reconstrucción de las bases éticas del enfoque de las capacidades de Amartya Sen [sitio en Internet]. [citado Dic 2009]. Disponible en: http://www-3.unipv.it/deontica/ca2004/papers/conill.pdf

11. Sen A. Desarrollo como libertad. Barcelona: Planeta; 2000.

12. Delgado P, Salcedo T. Aspectos conceptuales sobre los indicadores de Calidad de vida [sitio en Internet]. [citado Dic 2009]. Disponible en: http://www.webpondo.org/files oct dic 03/AspectosConceptuales.pdf

13. Saugstad A. La filosofía y la buena vida. PerSe [serie en Internet]. 2007 [citado Dic 2009];2(8). Disponible en: http://www.filosofia.mx/archivos/perse08.pdf

14. López T. Ética de la diferencia. En: Reyes R, editor. Diccionario Crítico de Ciencias Sociales. Terminología Científico-Social [sitio en Internet]. [citado Dic 
2009]. Disponible en:

http://www.ucm.es/info/eurotheo/diccionario/E/etica diferencia.htm

15. Ayerbe A, Cortés $P$, Medrano S. El desarrollo de los valores en la tercera edad: un estudio desde el marco cultural-contextual. Rev Psicodidáctica [serie en Internet]. 2001 [citado Dic 2009]. Disponible en: http://redalyc.uaemex.mx/redalyc/pdf/175/17501202.pdf

16. Fascioli A. Capacidades, perfeccionismo y autonomía del sujeto en el pensamiento de Amartya Sen. Actio [serie en Internet] Mar 2002 [citado Dic 2009];2(2). Disponible en: http://www.fhuce.edu.uy/public/actio/Textos/II2/Fascioli.pdf

17. Pereira G. Fundamentación universalista de una lista de capacidades requerida por la justicia global. Diánoia [serie en Internet] Nov 2006 [citado Dic 2009];LI(57). Disponible en: http://dianoia.filosoficas.unam.mx/info/2006/d57Pereira.pdf

18. Sen A. Desarrollo económico y libertad. La Factoría [serie en Internet] May-Dic 2006 [citado Dic 2009];XVI(30-31). Disponible en:

http://www.revistalafactoria.eu/articulo.php?id=359

19. Rivero C. ¿Calidad de vida en América Latina? Las reflexiones epistemológicas y teóricas que acompañan los procesos. Caracas: La Galaxia; 2000.

20. Gracia D. Ética de la Calidad de vida. En: Gracia D, editor. Ética y Vida 1: Fundamentación y enseñanza de la bioética. Santa Fé de Bogotá: Editorial El Buho; 1998.

21. Lolas F, Outomuro D. Bioética y salud pública. En: Lolas F, Quezada A, Rodríguez $E$, editores. Investigación en salud. Dimensión ética. Santiago de Chile: CIEB, Universidad de Chile; 2006. p. 103-7.

22. Cely G. La bioética en la sociedad del conocimiento. Santa Fé de Bogotá: 3R Editores; 1999.

23. Boff L. ¿Vivir mejor o el "buen vivir"? Koinonía. La Columna Semanal de Leonardo Boff No 321, 2009-03-27 [sitio en Internet]. [citado Dic 2009]. Disponible en: http://www.servicioskoinonia.org/boff/articulo.php?num=321

24. Humanismo y Conectividad. Frente a la complejidad del mundo: la simplicidad voluntaria [sitio en Internet]. [citado Dic 2009]. Disponible en: http://humanismoyconectividad.wordpress.com/2008/05/29/simplicidadvoluntaria/

25. Alguacil J. La Calidad de vida como síntesis de la complejidad. En: Calidad de vida y praxis urbana: nuevas iniciativas de gestión ciudadana en la periferia social de Madrid. Madrid: Centro de Investigaciones Sociológicas, Colección Monografías 179; 2006. p. 66-83 [sitio en Internet]. [citado Dic 2009]. Disponible en: http://habitat.aq.upm.es/cvpu/acvpu.pdf

26. Delgado C. Calidad de vida: una perspectiva latinoamericana. En: Boladeras $M$, Escobar J, Maldonado C, editores. Bioética y Calidad de vida. Bogotá: Ediciones EI Bosque; 2000. 
27. Morin E. Introducción al pensamiento complejo. Barcelona: Gedisa; 2003.

28. Morin E, Ciurana E, Mota R. Educar en la era planetaria. Barcelona: Gedisa; 2003.

29. Ugas G. La Complejidad: un modo de pensar. San Cristóbal, Venezuela:

Ediciones del Taller Permanente de Estudios Epistemológicos en Ciencias Sociales; 2006.

30. Morin E. El Método I. La naturaleza de la naturaleza. 5ta ed. Madrid: Ediciones Cátedra S.A.; 1999.

31. Bracho C, Román G, López J. Guía para la elaboración de proyectos de Trabajo de Especialización, Trabajo de Grado de Maestría y Tesis Doctoral. Valencia, Venezuela: Universidad de Carabobo; 2004.

32. Leal J. La Autonomía del Sujeto Investigador y la Metodología de Investigación. 2da ed. Valencia, Venezuela: Azul Intenso C.A.; 2009.

33. Martínez M. Postulados de la nueva racionalidad. En: Martínez M, editor. La Nueva Ciencia: Su Desafío, Lógica y Método. México: Editorial Trillas; 1999. p. 6988.

34. Soto M, Morin E. Complejidad y sujeto humano [tesis]. Universidad de Valladolid: Biblioteca Virtual Miguel de Cervamtes; 1999 [sitio en Internet]. [citado Dic 2009]. Disponible en:

http://www.archivochile.com/tesis/11 teofiloideo/11teofiloideo0004.pdf

35. Garciandía JA. Pensar sistémico. Una introducción al pensamiento sistémico. Bogotá: Editorial Pontificia Universidad Javeriana; 2005.

36. Capra F. El punto crucial. Buenos Aires: Editorial Estaciones; 1992.

Recibido: 26 de enero de 2010.

Aprobado: 6 de abril de 2010 .

Harold Guevara. Urbanización Prebo 2. Avenida 138, No 130-100. Valencia, estado Carabobo. Venezuela.

Teléf.: (58-241)-8239550. E-mail: hguevararivas@gmail.com 